\title{
Recurrence of Primary Breast Angiosarcoma 7 Years after Mastectomy in a 17-Year-Old Woman: A Case Report
}

\author{
Miaowei Wu ${ }^{a}$ Yajing Huang ${ }^{b}$ Wei Tian ${ }^{a} \quad$ Yihan Yao $^{a} \quad$ Yongchuan Deng $^{a}$ \\ ${ }^{a}$ Department of Surgical Oncology, the Second Affiliated Hospital, School of Medicine, Zhejiang University,

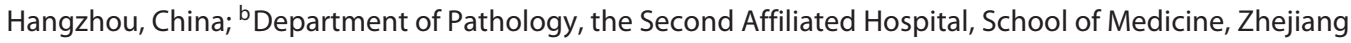 \\ University, Hangzhou, China
}

\section{Established Facts}

- Primary breast angiosarcoma affects patients usually at the age of 20-50 years, and, in previous studies, the follow-up period was not longer than 5 years.

\section{Novel Insights}

- Primary breast angiosarcoma can occur in patients less than 18 years old and should be followed up for more than 5 years.

Keywords

Breast · Angiosarcoma $\cdot$ Recurrence $\cdot$ Surgery

\section{Abstract}

Background: Primary breast angiosarcoma is a rare neoplasm; breast angiosarcoma patients under 20 years old are extremely rare. The prognosis is often poor due to a high relapse rate after surgical resection. Case Report: We report on a case of a 17-year-old woman suffering from a primary breast angiosarcoma, associated with the local recurrence of a right-breast angiosarcoma 86 months after mastectomy. She received extensive local excision and transplantation of an adjacent skin flap. The post-operation diagnosis was angiosarcoma of histological grade III. Conclusions: This is a rare case as the patient was below 18 years old and the follow-up was greater than 5 years. Taken together, long-term close follow-up is extremely important, regardless of how long after surgery and the status of the surgical margins.

(c) 2019 S. Karger AG, Basel

\section{Introduction}

Breast angiosarcoma is a highly aggressive vascular neoplasm and is estimated to constitute $0.05 \%$ of the malignant diseases of the breast [1]. Breast angiosarcomas can be divided into 2 types: primary breast angiosarcoma and secondary angiosarcoma resulting from the preceding breast cancer therapy. The causes of primary breast cancer remain unclear. Primary breast angiosarcomas are most common in women in their 30s and 40s [2]. Here, we present a case of primary breast angiosarcoma in a woman less than 18 years of age, with a follow-up period of over 5 years. The patient is still alive 8 months after her second resection, and no evidence of any local or distant disease is observable.

\section{KARGER}

(C) 2019 S. Karger AG, Basel
Yongchuan Deng

Department of Surgical Oncology, the Second Affiliated Hospital

School of Medicine, Zhejiang University

88 Jiefang Rd, Hangzhou 310009, Zhejiang, China

dyc001@zju.edu.cn 
a

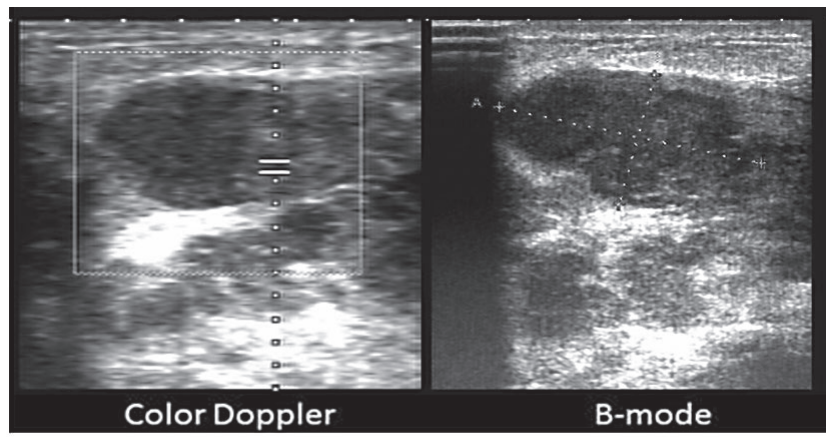

b

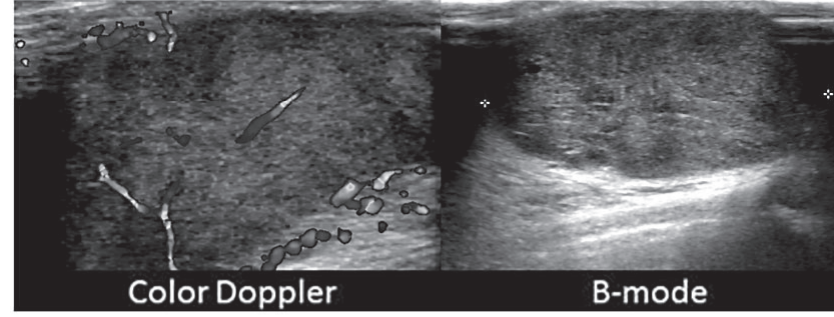

C

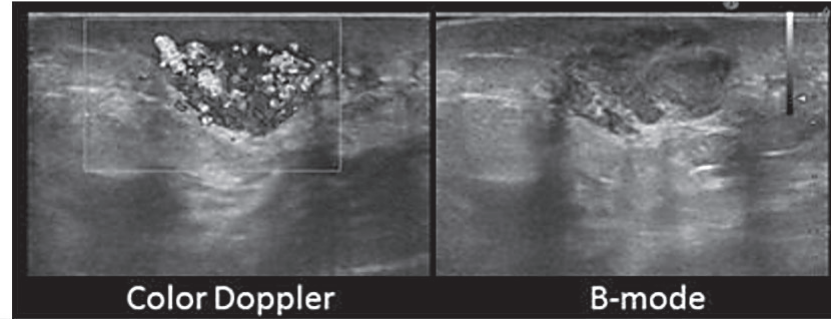

Fig. 1. Pictures of the sonographic appearance at presentation compared to the typical appearance. a The sonographic appearance at initial presentation, showing the tumor to be ill-defined, irregular in shape, and hypoechoic in B-mode (right), with absent color Doppler signals (left). b The sonographic appearance when the tumor recurred at the chest wall after 86 months, showing the mass to be well-defined, and hypoechoic in B-mode (right), with hypervascular Doppler signaling (left). c The typical appearance of a breast angiosarcoma, showing the tumor to be ill-circumscribed, irregular in shape, and hypoechoic in B-mode (right), with hypervascular Doppler signaling (left) [1].

\section{Case Report}

A 17-year-old woman was admitted to our hospital with a 6.0 $\times 5.5 \mathrm{~cm}$ lump in the upper quadrant of the right breast. No congenital vascular lesion was found in her breast before the breast angiosarcoma was diagnosed. Sonography showed the tumor to be ill-defined, irregular in shape, and hypoechoic in B-mode, with absent color Doppler signals (fig. 1). Histological findings of a needle biopsy specimen revealed the lesion to be an angiosarcoma. She underwent a mastectomy. The pathology findings of the gross specimen were consistent with grade III angiosarcoma. The tumor was observed to invade the surrounding adipose tissue. Endothelial markers (CD31, factor VIII), vascular markers (CD34, Ets-related gene (ERG), vascular endothelial growth factor (VEGF)), the tumor suppressor genes $P 53$ and $P 16$, and the mesenchymal marker vimentin were positively expressed in the tumor. We recommended adjuvant chemotherapy as a safety measure according to the clinical guidelines [3] and clinical studies on soft-tissue sarcoma [4-7]. However, she refused the option to receive adjuvant chemotherapy.
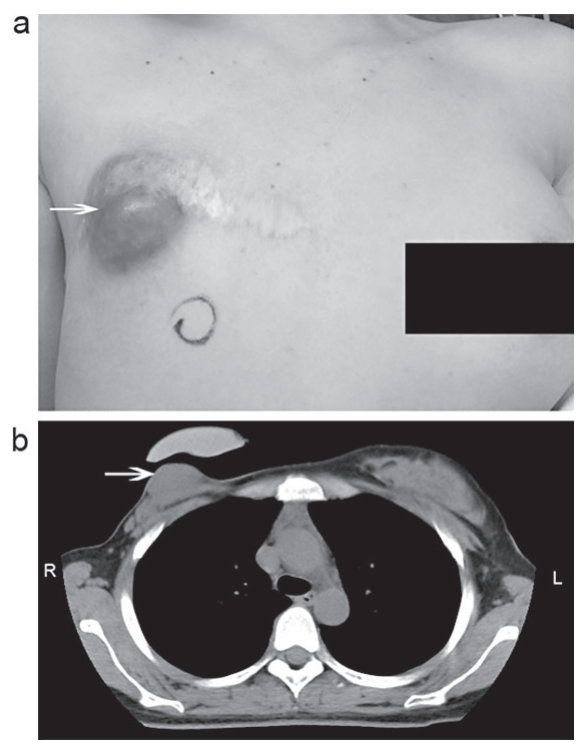

Fig. 2. Clinical presentation and CT scan after recurrence of the angiosarcoma. a Macroscopic appearance of the recurrence of primary breast angiosarcoma in the right chest wall (white arrows) 86 months after mastectomy. b Chest CT, done 86 months after mastectomy, showed a solid mass (white arrows) in the right chest wall.

After 86 months, the right chest wall showed a $5.5 \times 4.5 \mathrm{~cm}$ rapidly enlarging mass (fig. 2a). No axillary lymphadenopathy was palpated. A computed tomography (CT) scan of the chest showed a solid mass under the skin of the right chest wall (fig. 2b). Sonography showed the mass to be well defined and hypoechoic in Bmode, with hypervascular Doppler signaling. The patient underwent extensive local excision and transplantation of an adjacent skin flap.

Histopathological findings revealed a poorly differentiated soft-tissue angiosarcoma infiltrating into vessels and striated muscle. A papillary and capillary growth pattern formed by anastomosing vascular channels was observed (fig. 3a, b). Necrosis was also found in the tumor. Immunohistochemical examination showed positive expression of the endothelial marker CD31 (fig. 3c), the vascular markers CD34 (fig. 3d), factor VIII (fig. 3e), ERG (fig. 3f), and VEGF (fig. 3g), and of the tumor suppressor genes $P 53$ and P16. The mesenchymal marker vimentin was also positively expressed (fig. $3 \mathrm{~h}$ ). In contrast, the epithelial markers cytokeratin (CK) AE1/AE3, smooth muscle actin (SMA), and epithelial membrane antigen (EMA) were negatively expressed. The proliferation index Ki-67 was positively expressed at $25 \%$. After the second resection, we suggested the patient to undergo chemotherapy based on the current clinical guidelines [8], but she refused. The disease-free interval from the second resection to the publication of this case was 8 months, up until September of 2018.

\section{Discussion}

Breast angiosarcoma, a sarcoma arising from endothelial cells of blood vessels, occurs more often in women between 20 and 50 years [9]. However, the patient was 17 years old when first diagnosed. During physical examination, a painless mass with bluish skin discolorations was 

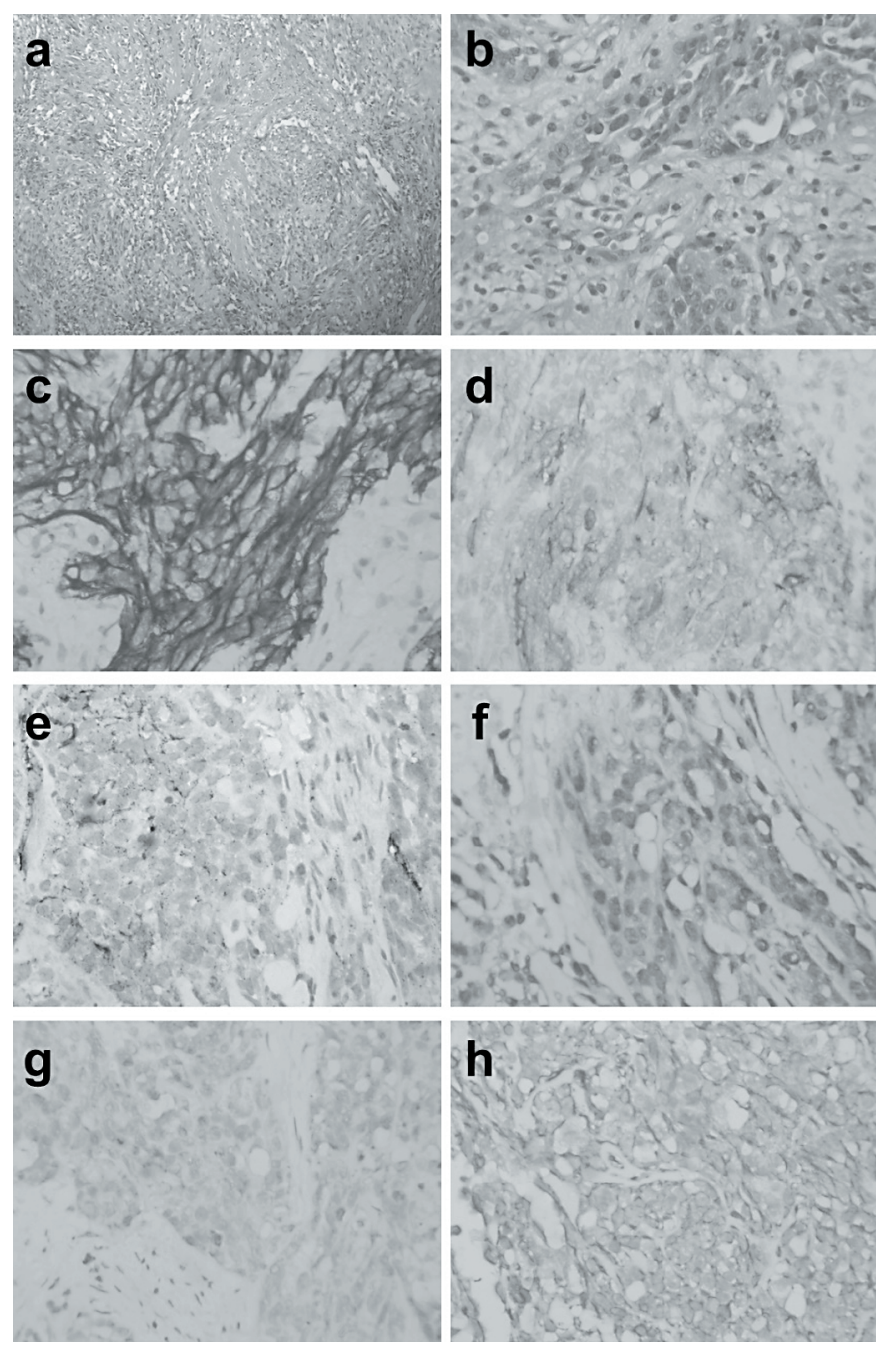

Fig. 3. Macroscopic findings of the resected specimen. Hematoxylin and eosin staining demonstrated a growth patterns of angiosarcoma present as ramifying irregular vascular structures, $\times 100$; $\mathbf{b}$ cytologically atypical endothelial cells with large hyperchromatic nuclei, high $\mathrm{N}: \mathrm{C}$ ratio, and mitotic figures, original magnification $\times 400$. Tumor stains positive for $\mathbf{c}$ CD31, $\mathbf{d}$ CD34, e factor VIII, $\mathbf{f}$ ERG, $\mathbf{g}$ VEGF, and $\mathbf{h}$ vimentin; $\mathbf{c}-\mathbf{h}$ original magnification, $\times 400$.

palpable, in accordance with the description in the literature [10]. Almost one-third of primary breast angiosarcomas can be determined by mammography [11], but our patient did not undergo mammography due to her young age. At the time of initial diagnosis, ultrasound revealed several honeycomb-like low-echo masses without blood flow in the right breast, which was different from the typical appearance of breast angiosarcoma [12]. However, when the condition recurred, the ultrasound demonstrated a mass with marked vascularity and unclear boundary, which was similar to the typical appearance.

Morphologically, growth patterns of angiosarcoma present as ramifying irregular vascular structures, including vasoformative growth, solid growth, papillary endothelial growth, and capillary-type growth forming anas- tomosing vascular channels. Atypical endothelial cells, including endothelial shapes that appear plumped, spindled, or epithelioid-like, have hyperchromatic nuclei of varying sizes. Necrosis and blood lakes may be found in the stroma. The diagnosis can be clarified by positive immunohistochemical expression of the endothelial biomarker CD31 in tumor cells, which is the most sensitive and specific indicator of angiogenic proliferation, while vascular markers such as CD34, factor VIII, D2-40, and Fli1 were also positively expressed [13]. Moreover, it is suggested that the proliferative index, $\mathrm{Ki}-67$, is a useful differentiating index when attempting to differentiate benign breast mesenchymal tumors from malignant ones [14]. It has been shown that neither preoperative fineneedle biopsy nor core needle biopsy is reliable enough, since a $37 \%$ false-negative rate has been reported [15]. Therefore, a surgical specimen for histological and immunohistochemical examination is necessary.

Surgery plays an indispensable role in the treatment of primary breast angiosarcoma [16]. Generally, mastectomy (including the muscular fascia) is recommended even in combination with postoperative radiotherapy. Lymphadenectomy is not carried out in the absence of clinical evidence of involvement [8]. Although, so far, there are no relevant clinical trials on breast angiosarcomas due to their rarity, it has been demonstrated that grade III primary breast angiosarcoma patients and patients with disease recurrence would benefit from chemotherapy [17]. The chemotherapy regimens refer to soft-tissue sarcomas. For high-risk patients, administering anthracyclines and ifosfamide for at least 3 cycles is an option [8]. In addition, doxorubicin, liposomal doxorubicin, and taxanes are possible choices for monotherapy [18]. As for targeted therapy, bevacizumab is an effective and well-tolerated agent against metastatic or locally advanced angiosarcoma [7]. Considering the high risk of local and systemic relapses, radiation may be used and the timing depends on the wound complications. The recommended regimen is a total dose of $50 \mathrm{~Gy}$ in fractions of 1.8-2 Gy, possibly with a boost of up to $66 \mathrm{~Gy}$, depending on the margins at the time of presentation and after resection. Furthermore, re-irradiation should be considered in radiation-induced angiosarcomas [8]. So far, there is no conclusive evidence that radiation increases the risk of secondary angiosarcoma.

In conclusion, primary breast angiosarcoma is a rare malignant sarcoma. It usually presents as a palpable, painless breast mass. Surgery is a necessary treatment option for the early stages of breast angiosarcoma. Chemotherapy and radiation are recommended to decrease the risk of recurrence. Long-term close follow-up is extremely important, regardless of how long after surgery or the status of the surgical margins. 


\section{Acknowledgements}

We would like to thank the anonymous reviewers who have helped to improve the paper. W.T. was supported by grants from the National Natural Science Foundation of China (grant no. 81502598) and the Zhejiang Provincial Natural Science Foundation of China (grant no. LQ14H160010). Y.D. was supported by grants from the Zhejiang Provincial Natural Science Foundation of China (grant no. LY14H160020).

\section{Statement of Ethics}

The authors have no ethical conflicts to disclose.

\section{Disclosure Statement}

None of the authors has conflicts of interest to disclose.

\section{References}

$\checkmark 1$ Hodgson NC, Bowen-Wells C, Moffat F, Franceschi D, Avisar E: Angiosarcomas of the breast: a review of 70 cases. Am J Clin Oncol 2007;30:570-573

-2 Iacoponi S, Calleja J, Hernandez G, Sainz de la Cuesta R: Primary breast angiosarcoma in a young woman. Int J Surg Case Rep 2016;24: 101-103.

-3 Demetri GD, Antonia S, Benjamin RS, et al.; National Comprehensive Cancer Network Soft Tissue Sarcoma Panel: Soft tissue sarcoma. J Natl Compr Canc Netw 2010;8:630674.

4 Tierney JF, Mosseri V, Stewart LA, Souhami RL, Parmar MK: Adjuvant chemotherapy for soft-tissue sarcoma: review and meta-analysis of the published results of randomised clinical trials. Br J Cancer 1995;72:469-475.

$\checkmark 5$ Maki RG: Role of chemotherapy in patients with soft tissue sarcomas. Expert Rev Anticancer Ther 2004;4:229-236.

6 Schlemmer M, Reichardt P, Verweij J, Hartmann JT, Judson I, Thyss A, Hogendoorn PC, Marreaud S, Van Glabbeke M, Blay JY: Paclitaxel in patients with advanced angiosarcomas of soft tissue: a retrospective study of the EORTC soft tissue and bone sarcoma group. Eur J Cancer 2008;44:2433-2436.
Agulnik M, Yarber JL, Okuno SH, von Mehren $\mathrm{M}$, Jovanovic $\mathrm{BD}$, Brockstein BE, Evens AM, Benjamin RS: An open-label, multicenter, phase II study of bevacizumab for the treatment of angiosarcoma and epithelioid hemangioendotheliomas. Ann Oncol 2013; 24:257-263.

$>8$ Casali PG, Abecassis N, Bauer S, et al.; ESMO Guidelines Committee and EURACAN: Soft tissue and visceral sarcomas: ESMO-EURACAN Clinical Practice Guidelines for diagnosis, treatment and follow-up. Ann Oncol 2018;29(suppl 4):iv268-iv269.

9 Scow JS, Reynolds CA, Degnim AC, Petersen IA, Jakub JW, Boughey JC: Primary and secondary angiosarcoma of the breast: the Mayo Clinic experience. J Surg Oncol 2010;101: 401-407.

10 Aljohani B, Al-Twajeri T, Alameer A, Alzaydi T, Alawwad S, Anwar I, Alshabanh M, Tulba A, Almalik O: Clinicopathological features of breast angiosarcoma: a 16-years single-institution experience. Int J Surg Case Rep 2017; 37:211-215.

11 Bennani A, Chbani L, Lamchahab M, Wahbi M, Alaoui FF, Badioui I, Melhouf MA, Amarti A: Primary angiosarcoma of the breast: a case report. Diagn Pathol 2013;8:66.

-12 Tanaka Y, Uchida A, Umemoto T, Morishima I, Kikuchi K, Tohno E, Ueno E: Spontaneous regression of breast angiosarcoma after conservative treatment with radiotherapy: a case report and review of the literature. J Med Ultrason (2001) 2015;42:427-432.
13 Boufettal H, Noun M, Hermas S, Samouh N, Benayad S, Karkouri M, Zamiati S: Breast angiosarcoma: a case report (Article in French). Ann Pathol 2013;33:217-220.

14 Shin SJ, Lesser M, Rosen PP: Hemangiomas and angiosarcomas of the breast: diagnostic utility of cell cycle markers with emphasis on Ki-67. Arch Pathol Lab Med 2007;131:538544.

15 Costa S, Graca SA, Ferreira A, Maciel J: Breast angiosarcoma secondary to phyllodes tumour. BMJ Case Rep 2012;2012:pii: bcr2012007545.

16 Keshav P, Hegde SS: Bilateral primary angiosarcoma of the breast. Case Rep Surg 2013; 2013:139276.

17 Sher T, Hennessy BT, Valero V, Broglio K, Woodward WA, Trent J, Hunt KK, Hortobagyi GN, Gonzalez-Angulo AM: Primary angiosarcomas of the breast. Cancer 2007;110: 173-178.

18 D’Angelo SP, Munhoz RR, Kuk D, Landa J, Hartley EW, Bonafede M, Dickson MA, Gounder M, Keohan ML, Crago AM, Antonescu CR, Tap WD: Outcomes of systemic therapy for patients with metastatic angiosarcoma. Oncology 2015;89:205-214. 